\title{
THE EFFECT OF VORTEX FINDER SHAPE ON SEPARATION EFFICIENCY AND PRESSURE DROP IN LOWER-STAGE CYCLONES USED FOR CLINKER BURNING. A CFD STUDY
}

\author{
M. Wasilewski*, G. Ligus**
}

\begin{abstract}
This study analyses the effect of the shape of the vortex finder on separation efficiency and pressure drop in low-stage cyclones used for clinker burning. The CFD method was applied. The RANS and RSM models were used to simulate turbulent flow. Six variants of cylindrical and conical vortex finders were analysed. The results indicate that in the case of low-stage cyclones (where separation efficiency is not the main objective function) it is reasonable to use a cylindrical vortex finder.
\end{abstract}

Keywords: cyclone separator, CFD, pressure drop, separation efficiency, suspension preheater

\section{Introduction}

The application of the dry method with external cyclone suspension preheater has considerably affected the energy consumption of clinker manufacturing. In addition to greatly reducing fuel consumption, the method has also allowed for the introduction of two-stage rotary kiln heating, i.e., initial decarbonisation. Modern installations consist of 4-6 stages. The operation of a cyclone suspension preheater has been explained in such studies as (Wasilewski et al., 2016, Kashani et al., 2018). The structure of cyclone separators of each stage in a preheater depends on their function and temperature conditions. First-stage cyclone separators should maximise separation efficiency, whereas low-stage cyclones, due to the progressively smaller size of particles (decarbonisation) and low gas density (high temperature), should minimise pressure drop (while maintaining a separation efficiency of $>70 \%$ ). Furthermore, low-stage cyclones frequently suffer from deposit build-ups due to the circulation of alkali in the process.

A review of studies on the subject indicates that most of them focus on traditional cyclone separators. Few studies address cyclones used for clinker burning (e.g. Mikulcic et al., 2014, Wasilewski et al., 2016, 2017, Mariani et al., 2017, Kashani et al., 2018), and those that do usually concern first-stage cyclone separators. This motivated the authors of this study to investigate the effect of the shape of the vortex finder on separation efficiency and pressure drop in cyclone separators within the lower stages of a suspension preheater.

\section{Materials and methods}

The computational fluid dynamics (CFD) method was applied. The $3 \mathrm{~d}$ model was created based on the real geometry (used in a cement plant) of a third-stage cyclone separator (Figure 1a). During previous modernisation works, the vortex finder was removed from the cyclone due to deposit build-ups and in order to reduce pressure drop. However, the modification resulted in an excessive drop in separation efficiency. Heat and flow parameters of the current installation were measured to allow for the validation of the applied numerical assumptions and the introduction of real boundary conditions (Table 1).

Marek Wasilewski, PhD.: Faculty of Production Engineering and Logistics, Opole University of Technology, 76 Proszkowska St., 45-758 Opole, Poland, m.wasilewski@ po.opole.pl

** Grzegorz Ligus, PhD.: Faculty of Mechanical Engineering, Opole University of Technology, 76 Proszkowska St., 45-758 Opole, Poland, g.ligus@po.opole.pl 
Subsequently, the authors proposed six structural solutions involving various configurations of the vortex finder (Figure 1b): a cylindrical vortex finder with three different lengths and a conical vortex finder with three different lengths, which are the most popular types of vortex finders in cyclone separators.

Flow was analysed based on the finite volume method using the Ansys Fluent 18.2 software package. The Reynolds Average Navier-Stokes (RANS) method and the Reynolds Stress Model (RSM) were used to simulate turbulent flow. Studies such as (Wang et al., 2006, Wasilewski et al., 2016, 2017, Pei et al., 2017, Kashani et al., 2018) provide detailed information on both RANS and RSM. The selected approach of solving was based on the statement of pressure-based pressures equations (Pressure Based Solution Method Segregated Solver). The solution of the differential equations utilized the SIMPLE algorith. The second-order upwind interpolation method was used to determine the representative samples of the constituent values on the surface of the control volumes. The condition of convergence for the continuity equation was set at $10^{-6}$, whereas for the remaining equations, it was $10^{-3}$. The presence of the multiphase flow was simulated using the Euler-Lagrange method. In this case, fluid is considered as a continuous phase, whereas the dispersed phase (solid phase) is identified through the particle tracking. The description of this method can be found in studies such as (Wang et al., 2006, Wasilewski et al., 2016, 2017, Pei et al., 2017, Kashani et al., 2018). A hexagonal mesh with a varying density, depending on the area of the device, was used to discretise the computational domain. To total number of mesh elements ranged from about 840,000 to about 855,000 depending on the variant.
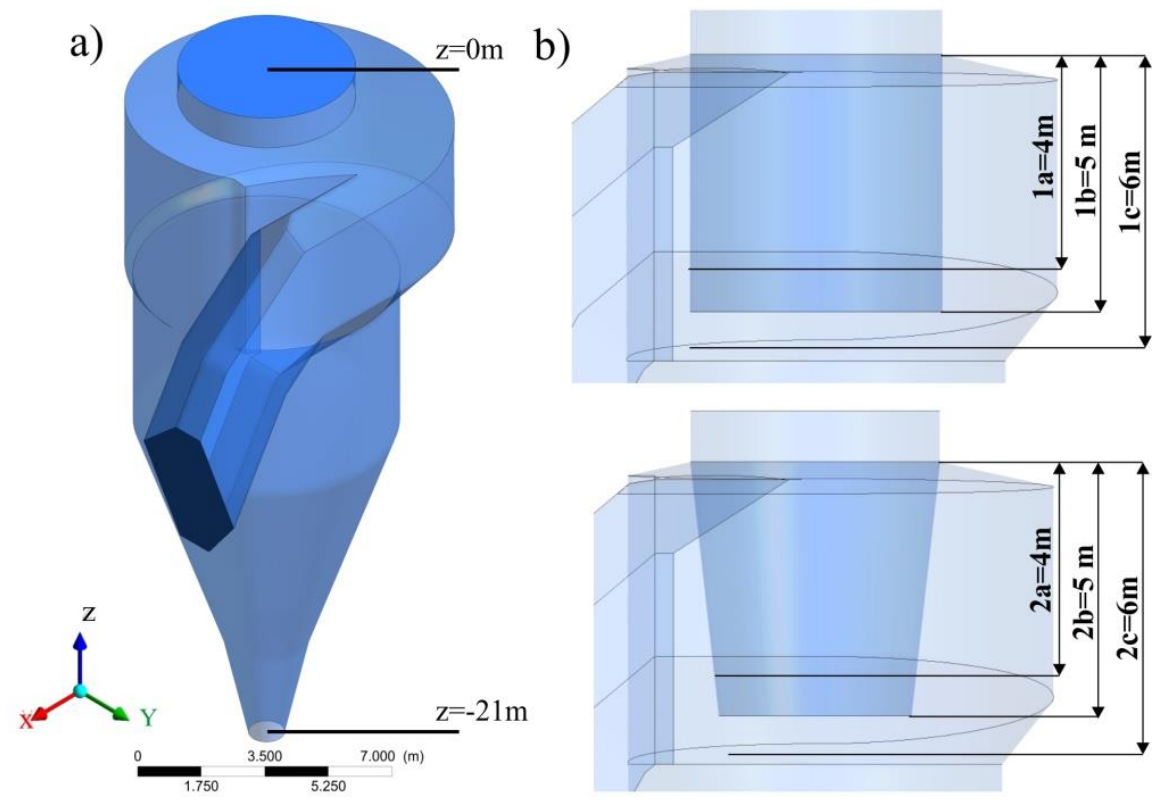

Fig. 1: a) Geometry of the base cyclone separator, b) Variants of the applied modifications.

Tab. 1: Operating conditions used in the simulation.

\begin{tabular}{cc}
\hline Inlet & Inlet velocity $29.8 \mathrm{~m} / \mathrm{s}$ \\
\hline Outlet & Outflow \\
\hline The walls of the cyclone & The standard walls function \\
\hline Discrete phase model & One-way coupling \\
\hline Temperature of the gas phase & $1220 \mathrm{~K}$ \\
\hline Density of gas; Density of a particle & $0.297 \mathrm{~kg} / \mathrm{m}^{3} ; 2700 \mathrm{~kg} / \mathrm{m}^{3}$ \\
\hline
\end{tabular}

\section{Results and discussion}

The pressure drop in the numerical model was validated with respect to the base variant (the current geometry in cement plant). Operational pressure drop amounted to about $1200 \mathrm{~Pa}$, and pressure drop obtained based on CFD amounted to $1210 \mathrm{~Pa}$. A very small computational error indicated that the numerical research was configured correctly. A lack of a precise measurement of the real installation due to the parameters of the two-phase mixture (e.g. high temperature) made it impossible to validate separation efficiency. 
Figure 2 presents the obtained values of pressure drop (a) and separation efficiency (b) for all analysed variants. The data indicate that the shape of the vortex finder had a major effect on pressure drop. A cylindrical vortex finder provided a considerably lower pressure drop than a conical one, regardless of length. Thus, the application of a cylindrical vortex finder may bring considerable savings in power consumption incurred by the fans of the preheater tower. The lowest pressure drop (1620 Pa) was obtained for a cylindrical vortex finder with a length of $4 \mathrm{~m}$. The lowest pressure drop among conical vortex finders $(2293 \mathrm{~Pa}$ ) was also obtained for the shortest vortex finder. For both shapes, pressure drop increased with an increase in length.
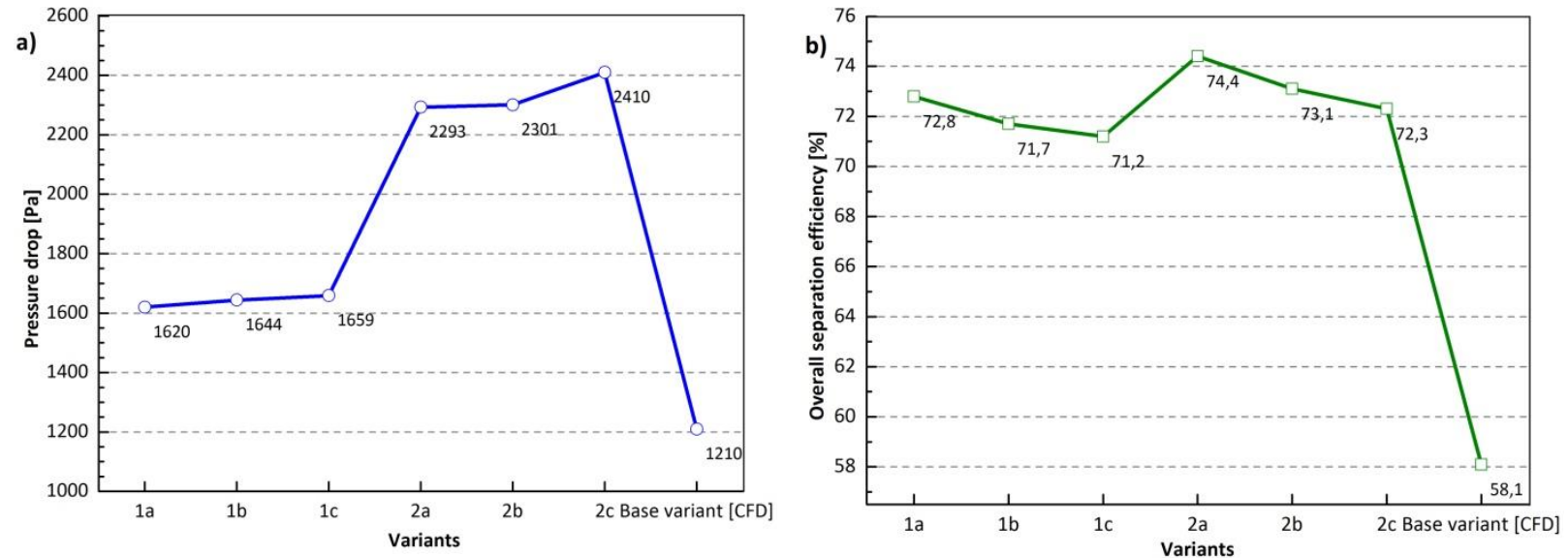

Fig. 2: Pressure drop (a) and overall separation efficiency (b) for various option of modification.

The obtained results were very similar for separation efficiency. The highest separation efficiency (74.4\%) was obtained for a conical vortex finder with a length of $4 \mathrm{~m}$, and the lowest separation efficiency (71.2\%) was obtained for a cylindrical vortex finder with a length of $6 \mathrm{~m}$. For both shapes, separation efficiency decrease with an increase in length. However, the obtained values of separation efficiency, regardless of shape, were much higher than those obtained for the base variant using CFD $(58.1 \%)$.

Figure 3 presents the fractional separation efficiency for each diameter of solid particles. These data confirm the trend that was observed for overall separation efficiency in each variant. The lowest differences were observed for particles with a diameter of $2.5 \mu \mathrm{m}$ (about $2 \%$ regardless of the variant, including the base variant without a vortex finder). This confirms the thesis that such particles are not separated in mechanical dust collectors of this type.

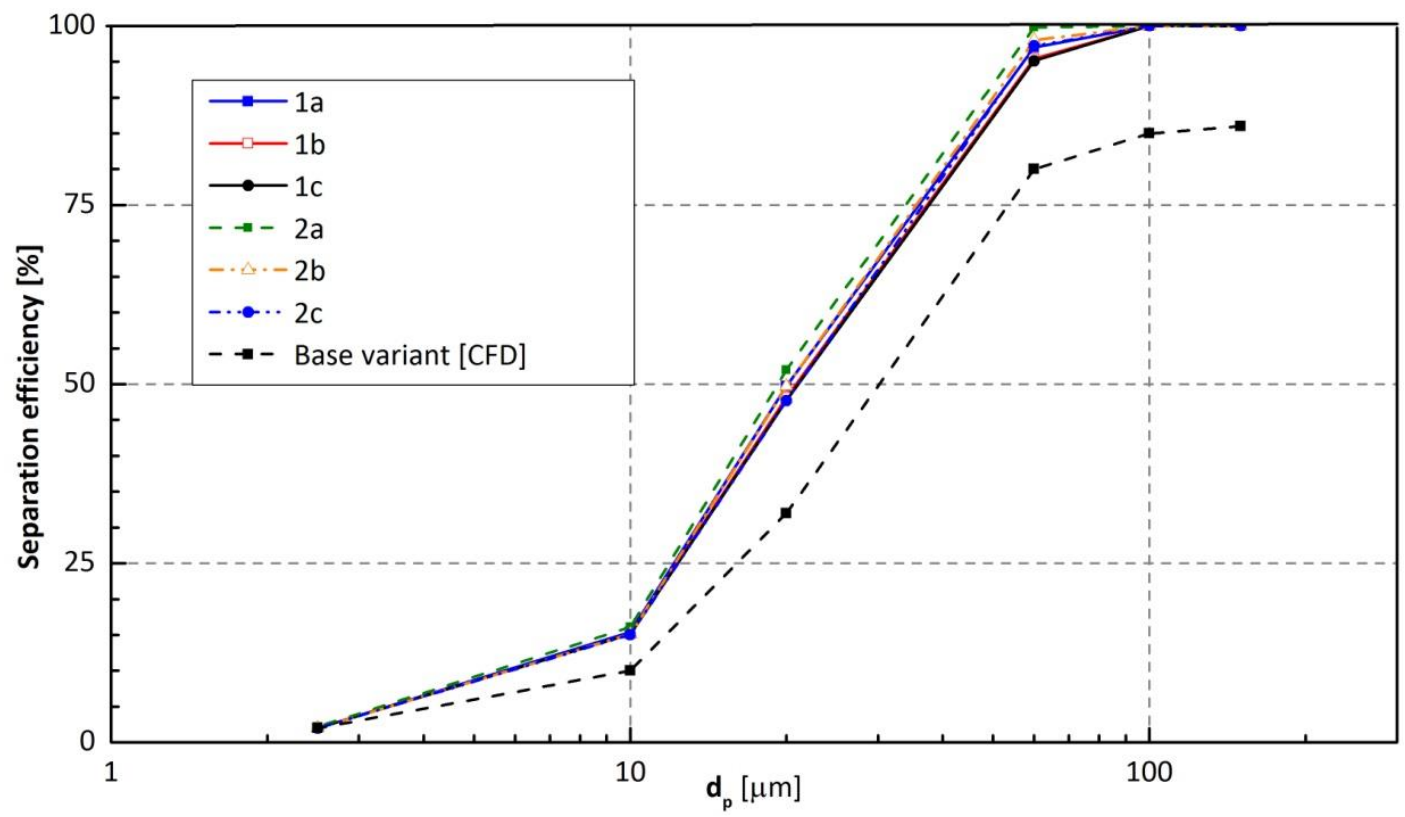

Fig. 3: Separation efficiency of each variant according to particle diameter. 
Figure 4 presents the values of tangential velocity (one of the primary parameters in the analysis of flow in cyclone separators) at a height of $\mathrm{z}=-6.5 \mathrm{~m}$. The obtained distributions are typical for cyclone separators. The lowest values were located in the axis - this is due to the occurrence of a upward inner ascending vortex in this region. The application of a vortex finder increased tangential velocities by increasing flow turbulence near the vortex finder. A cylindrical vortex finder helped to limit this phenomenon, but at the cost of a lower separation efficiency.

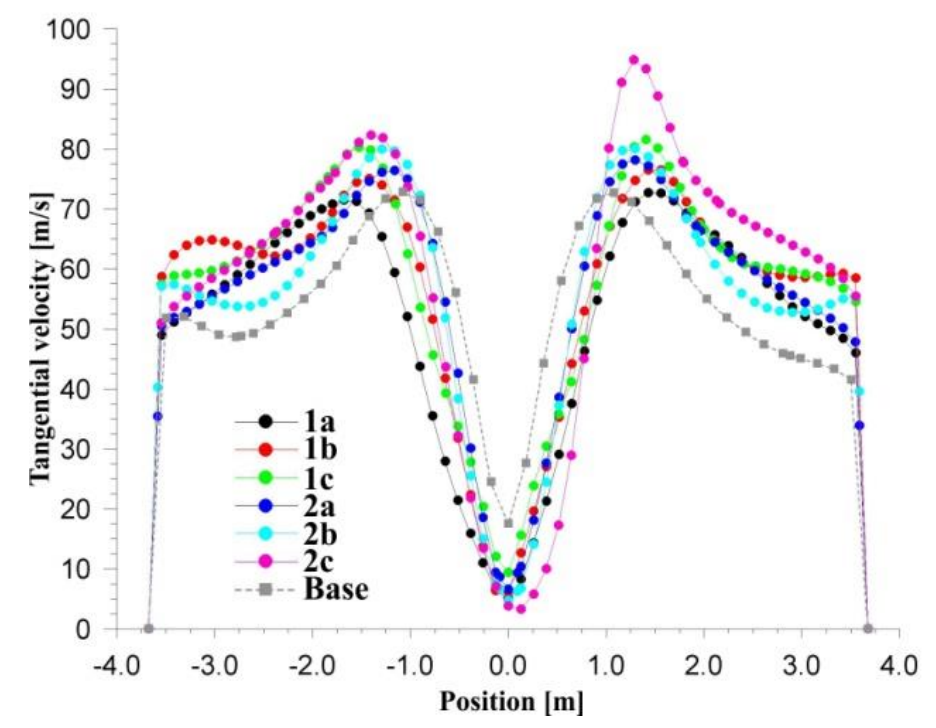

Fig. 4:Profiles of tangential velocity in the location $z=-6.5 \mathrm{~m}$.

\section{Conclusions}

The conducted analysis of the flow of a two-phase mixture near the vortex finder allows for the conclusion that the introduction of even technically simple and inexpensive modifications may improve the efficiency of an installation. While the removal of the vortex finder helped reduce pressure drop in the studied installation, it also severely reduced separation efficiency. The solutions proposed in this study allow for a considerable improvement in separation efficiency. The results indicate that in the case of low-stage cyclones (where separation efficiency is not the main objective function) it is reasonable to use a cylindrical vortex finder than a conical vortex finder, as the application of the latter leads to a considerable pressure drop with only a slight increase in separation efficiency.

\section{Acknowledgement}

This research was carried out with the support of the Interdisciplinary Centre for Mathematical and Computational Modelling (ICM) University of Warsaw under grant no G71-5.

\section{References}

Kashani, E., Mohebbi, A. and Heidari, M.G. (2018) CFD simulation of the preheater cyclone of a cement plant and the optimization of its performance using a combination of the design of experiments and multi-gene genetic programming. Powder Technology, 327, pp. 430-441.

Mariani, F., Risi, F. and Grimaldi, C. (2017) Separation efficiency and heat exchange optimization in a cyclone. Separation and Purification Technology, 179, pp. 393-402.

Mikulcic, H., Vujanovic, M., Ashhab, M.S. and Duic, N. (2014) Large eddy simulation of a two-phase reacting swirl flow inside a cement cyclone, Energy 75, pp. 89-96.

Pei, B., Yang. L., Dong, K., Jiang, Y., Du, X. and Wang, B. (2017) The effect of cross-shaped vortex finder on the performance of cyclone separator. Powder Technology 313, pp. 135-144.

Wang, B., Xu, D.L., Chu, K.W. and Yu, A.B. (2006) Numerical study of gas-solid flow in a cyclone separator. Applied Mathematical Modelling, 30, pp. 1326-1342.

Wasilewski, M. and Duda, J. (2016) Multicriteria optimisation of first-stage cyclones in the clinker burning system by means of numerical modelling and experimental research. Powder Technology, 289, pp. 143-158.

Wasilewski, M. and Brar, L.S. (2017) Optimization of the geometry of cyclone separators used in clinker burning process: A case study. Powder Technology, 313, pp. 293-302. 